\title{
Stable immobilization of size-controlled bimetallic nanoparticles in photonic crystal fiber microreactor
}

Sebastian Ponce, ${ }^{1}$ Macarena Munoz, ${ }^{1}$ Ana M. Cubillas, ${ }^{2}$ Tijmen G. Euser, ${ }^{2,3}$ Gui-Rong Zhang, ${ }^{1}$ Philip St. J. Russell, ${ }^{2}$ Peter Wasserscheid, ${ }^{4}$ Bastian J.M. Etzold ${ }^{* 1}$

${ }^{1}$ Technische Universität Darmstadt, Ernst-Berl-Institut für Technische und Makromolekulare Chemie, 64287 Darmstadt, Germany;

${ }^{2}$ Max-Planck Institute for the Science of Light, Guenther-Scharowsky-Str. 1/Bldg. 24, 91058

Erlangen, Germany;

${ }^{3}$ NanoPhotonics Centre, Cavendish Laboratory, University of Cambridge, J. J. Thomson

Avenue, Cambridge CB3 0HE, United Kingdom;

${ }^{4}$ Lehrstuhl für Chemische Reaktionstechnik, University of Erlangen-Nuremberg, 91058

Erlangen, Germany;

*Corresponding author: Prof. Bastian J.M. Etzold

$$
\begin{aligned}
& \text { Tel.: +49 (6151) } 1629983 \\
& \text { Fax: +49 (6151) } 1629982
\end{aligned}
$$

E-mail: etzold@tc1.tu-darmstadt.de 


\begin{abstract}
The possibility of immobilizing ex situ synthesized colloidal bimetallic nanoparticles (NPs) of well-defined characteristics inside hollow core photonic crystal fiber (HC-PCF) microreactors is demonstrated. With our developed method, PtNi clusters with average particle sizes ranging from 4.4 to $8.3 \mathrm{~nm}$ remain strongly-attached to the fiber core, and can be used as active catalysts for the hydrogenation of an azobenzene dye. The study revealed that optical transmission exhibits a size-dependent behavior, i.e., the smaller the NPs always bring in less optical signal loss. Sufficient light transmission was achieved for all particle sizes and furthermore, these catalytic PCF microreactors allowed to obtain kinetic data with a $10^{6}$ times lower amount of precious metals than that of a conventional batch reactor. This new generation of PCF microreactors opens up an unprecedented pathway for in situ catalyst screening.
\end{abstract}

Keywords: heterogeneous catalysis, in situ UV/Vis spectroscopy, microreactors, photonic crystal fibers, PtNi nanoparticles 


\section{Introduction}

Catalysis is the cornerstone of many processes in the chemical, pharmaceutical, and energy industries [1]. Due to its key importance, novel strategies are required to enhance the efficiency and sustainability of catalytic technologies. In this context, the study of catalysts under operation is an important tool for their optimization that could lead to reduced operating costs, and therefore has received much attention in the last years [2]. Microreactors provide a new platform for catalyst screening, as they offer unprecedented advantages such as minimal sample consumption, improved safety, enhanced mass transfer, and precise control of reaction parameters and conditions [3-5]. Recently, the integration of optical waveguides and microfluidic channels led to the new field of optofluidics, which has found a wide range of applications in biology and chemistry [6, 7]. In particular, optofluidic microreactors are especially interesting for catalysis, as they allow precise control of light and fluid sample, enable in situ detection, and could enhance the detection sensitivity and photocatalytic activity [8].

Our group recently developed a new concept of optofluidic microreactor based on a hollow-core photonic crystal fiber [9, 10]. HC-PCFs are optical fibers that consist of a micron-scale central hollow core surrounded by a cladding formed by an array of channels running along the entire length of the fiber (Fig. 1a) [11, 12]. This configuration uniquely allows single-mode and low-loss propagation of light in a tiny liquid-filled core. The resulting optofluidic microreactors offer sample volumes that can be as small as a few nanoliter per cm interaction length, while the strong confinement in the core maximizes the interaction of light with the sample. Furthermore, samples can be easily introduced into the hollow channels without the cumbersome post-processing techniques typically needed in conventional fiber sensors [10, 13]. Importantly, the system also allows in situ reaction monitoring by absorption spectroscopy, with sensitivities not attainable with conventional systems. These unique 
features have motivated the use of various types of HC-PCFs as highly-sensitive chemical sensors [14] and microreactors for photo-chemistry [15-18] and catalysis [9, 19].

In a former contribution, we proved that our HC-PCF can be turned into a heterogeneous catalytic microreactor [20]. This was achieved by developing a technique based on wetimpregnation with salt solutions to in situ grow catalyst nanoparticles on the walls of the fiber [9]. This process allowed the uniform deposition of Rh NPs over extended lengths of the HCPCF microreactor. The NPs obtained ranged from 25 to $120 \mathrm{~nm}$ in mean diameters, proportional to the amount of $\mathrm{Rh}$ precursor concentration used in the impregnation process. Unfortunately, this approach did not enable to obtain particles in the range of interest below $10 \mathrm{~nm}$ or the precise control of the catalyst particle characteristics such as particle size and shape, while both are particularly important for structure-sensitive reactions such as catalytic hydrogenations [21]. Furthermore, although the reaction kinetics were monitored online, significant optical losses were obtained $\left(16 \mathrm{~dB} \mathrm{~m}^{-1}\right.$ at $488 \mathrm{~nm}$ for $1 \%$ surface coverage and 25 nm particle size). Alternatively, recent works have also demonstrated the successful immobilization of metal NPs (e.g., Au and Ag) on the inner walls of various types of PCFs for surface-enhanced Raman scattering applications [22, 23]. However, the resulting fibers showed high losses or modified light guidance properties, mainly due to the big size of the NP clusters (40-200 nm). In this sense, the development of novel nanocatalysts of small and controlled size is crucial, in order to reduce scattering losses inside HC-PCFs.

Here, those limitations are overcome by incorporating ex situ synthesized small-sized colloidal NPs of well-defined properties inside HC-PCFs. In contrast to conventional methods of catalyst preparation, the synthesis of colloidal NPs allows high control of the shape and size of the NPs within a narrow distribution, which could help to identify correlations between the NP structure and its catalytic activity [24, 25]. At the same time, the immobilization of small-sized NPs on the core walls of the HC-PCF, away from the guided light at the center of the core, would prevent the impairment of the excellent light guidance properties of the fibers. 
As an actual research direction in catalysis is employing combinations of noble (e.g. Pd, Rh, Pt) and non-noble (e.g. Fe, Ni) metals in nanoalloyed NPs, which can improve activity and stability of conventional monometallic catalysts and reduce their production costs [26-28], this work focuses on bimetallic NPs. From the possible combinations, bimetallic PtNi was chosen, which has been previously been successfully applied to several hydrogenation reactions [29-31], and their superior performance compared to their monometallic counterparts is well proved [32].

Thus, the aim of this work is to develop a method for the deposition of ex situ synthesized bimetallic PtNi NPs of well-defined characteristics inside HC-PCFs to obtain catalytic HCPCF microreactors. Special focus is given in a reproducible loading and a long term stable immobilization of the NPs. The performance of the resulting HC-PCF microreactors will be investigated in a model structure-sensitive reaction such as the catalytic hydrogenation of azobenzene. The azobenzene dye N-ethyl-N-(2-hydroxyethyl)-4-(4-nitrophenylazo)aniline, commercially known as Disperse Red 1 (DR1), was chosen as target pollutant. In this case, the hydrogenation of azo dyes results in the breakdown of the chromophore [33], and therefore to a decrease in absorption over time of the $\pi-\pi^{*}$ transitions, which can be in situ monitored using absorption spectroscopy in the visible wavelength range.

\section{Experimental Section}

\subsection{PtNi NPs synthesis}

The size-controlled bimetallic NPs, with average size ranging from 4.4 to $8.3 \mathrm{~nm}$, were synthesized as a co-reduction of Pt and Ni following a solvothermal method [34]. Briefly, acetylacetonate salts of both metals and the capping agent PVP were dissolved in a mixture of benzyl alcohol and aniline for 15 min under stirring at room temperature. Afterwards, the solution was introduced in a PTFE-lined vessel and heated at temperatures within the range of 150-190 ${ }^{\circ} \mathrm{C}$ for different reaction times (6-15 h). The concentration of precursor salts, capping 
and reducing agents was varied in order to adjust the average size of the NPs. Once the reactor was cooled, the colloidal NPs were precipitated by adding an excess of acetone (1:3, $\mathrm{v} / \mathrm{v}$ ) to the reacted solution. In the last step, the NPs were purified by dispersion in a solution of ethanol/acetone (50-50\%, v/v) by sonication, followed by centrifugation (6000 rpm during $10 \mathrm{~min}$ ) and removal of the supernatant, obtaining a sludge of PtNi NPs redispersible in water and polar solvents.

\subsection{Hollow core photonic crystal fibers}

A kagomé-lattice HC-PCF was used (see Fig. 1a). The fiber has a 25- $\mu$ m hollow core surrounded by a cladding microstructure consisting of around 100 channels of $12 \mu \mathrm{m}$ diameter each (overall cladding diameter $140 \mu \mathrm{m}$ ). The fiber was particularly designed inhouse to offer broadband guidance in the 600-1000 nm range, when air filled. The transmission window shifts to 300-600 nm when both core and cladding holes are filled with isopropanol $(n=1.36)[10,35]$ and the fiber shows minimum losses of around $1 \mathrm{~dB} \mathrm{~m}^{-1}$ (at 900 $\mathrm{nm})$. The mode intensity profile at the fiber output shows that the guided light is indeed wellconfined in the liquid-filled core, when both core and cladding holes are filled. In this case, most of the light is guided in the fundamental mode in the core (right inset Fig. 1c). The typical fiber length used was $L=35 \mathrm{~cm}$, corresponding to a sample volume of just $140 \mathrm{~nL}$ in the hollow core, and $3000 \mathrm{~nL}$ in the cladding channels.

\subsection{PtNi NPs deposition in HC-PCFs}

For initial optical loss experiments, size-controlled colloidal PtNi NPs were deposited onto the core of HC-PCFs following a similar procedure previously reported [20]. First, the cladding microstructure of the fiber was collapsed using a fusion splicer in order to deposit the NPs only in the core of the fiber. In this way the introduction of NPs in the small cladding channels is avoided and the excellent light guidance properties of the fiber is not hindered. Then, impregnation solutions of PtNi NPs in isopropanol at different concentrations $(0.1-3 \times$ 
$10^{17} \mathrm{NPs} \mathrm{L}^{-1}$ ) and sizes (4.4, 6.3 and $8.3 \mathrm{~nm}$ ) were pumped into the core of the HC-PCF using a pressurized reactor until the first droplet of impregnation solution appeared on the open side of the fiber (approx. impregnation time $\leq 3 \mathrm{~s}$ ). Afterwards, the solvent was evaporated for 2 hours $\left(120^{\circ} \mathrm{C}\right.$, sonication) under vacuum conditions (10 mbar), resulting in the attachment of PtNi NPs to the inner fiber core wall. In addition, to prepare reliable catalytic HC-PCF microreactors for long-term experiments, the influence of the impregnation time (varied from a few seconds to 15 min using a $6.3 \mathrm{~nm}$ NP impregnation solution with a concentration of 1.2 $\times 10^{17} \mathrm{NPs} \mathrm{L}^{-1}$ ) as also the stability of the immobilized clusters during flushing experiments was tested.

\subsection{Experimental setup for the hydrogenation of DR1 in HC-PCF (Fig. 1c):}

Samples of $1 \mu \mathrm{M}$ DR1 dissolved in isopropanol were delivered to the HC-PCF via a pressurized reactor through a capillary system. The fiber ends were introduced into custommade liquid cells, surrounded and fixed by standard PEEK tubing connectors [10]. A flow meter was used to monitor the flow through the system. Supercontinuum light (from 475 to $2200 \mathrm{~nm}$ ), generated by a $1064 \mathrm{~nm}$ pump laser and a 20-m-long endlessly-single mode (ESM) PCF, was used as source in the system. Light was in- and out-coupled to the liquid-filled kagomé HC-PCF using standard microscope objectives. A beam splitter (BS) was used to reflect a small fraction of the light transmitted through the HC-PCF on to a charge coupled device (CCD) camera, which measured the irradiance profile of the guided mode. The remainder of the light was coupled into an optical spectrum analyzer (OSA), monitoring the changes in absorption. The time-evolution of DR1 was monitored on line by optical absorption spectroscopy at $510 \mathrm{~nm}$.

((Figure 1)) 


\section{Results and discussions}

\subsection{Nanoparticles characterization and catalytic behavior}

Fig. 1b shows the representative TEM image of $6.3 \mathrm{~nm}$ PtNi NPs (see Fig. S1 in the SI for TEM images and particle size histograms of the NPs at different sizes). The obtained NPs showed a well-defined truncated octahedral shape in a mixed PtNi nanoalloy, confirmed by the measured interplanar distance [34].

The catalytic activity of monometallic Pt and bimetallic PtNi NPs in the hydrogenation reaction of DR1 was firstly studied using a conventional stirred-tank reactor (see SI for experimental details). A clear positive synergic effect between Pt and Ni metals was observed, as PtNi-12 nm showed in the hydrogenation of DR1 almost a 3 times higher reaction kinetic constant, compared to Pt-12.4 nm NPs (see Fig. S2 in the SI). Furthermore, the effect of $\mathrm{H}_{2}$ pressure, concentration, and size of PtNi NPs on the hydrogenation of DR1 was also investigated. Data on the catalyst concentration influence is given in Fig. 2. The degradation of DR1 was appropriately fitted by a reaction model being second order for DR1. As a pressure variation was showing only a minor effect on the kinetics in the range studied in this work (1-10 bar, Fig. S3) a zero order for $\mathrm{H}_{2}$ was employed in the model. Interestingly, the reaction rate was observed to clearly depend on the size of the NPs (Fig. S4), which indicates that the DR1 hydrogenation is indeed a structure-sensitive reaction. The activity exhibits a volcano-dependent behavior on the NP sizes, with the maximum activity obtained at $6.3 \mathrm{~nm}$. (see Fig. S5 in the SI).

((Figure 2))

\subsection{Optical losses}

In first immobilization experiments the influence of the NP deposition on resulting optical transmission of the fiber was investigated. For the immobilization the standard method described in the experimental section was employed. Furthermore, the PtNi NPs size was 
varied as also the NP concentration in the impregnation solution $\left(0.1\right.$ to $\left.3 \times 10^{17} \mathrm{NPs} \mathrm{L}^{-1}\right)$. The loss introduced by the NPs $\left(\mathrm{dB} \mathrm{m}^{-1}\right)$ was measured using the conventional cut-back technique method detailed in the SI and the results are shown in Fig. 3. In all cases, the resulting HCPCFs were proved to maintain their excellent guidance properties, even at high NP loadings, and light was guided in a fundamental mode at the central core (right inset Fig. 1c). As expected, the average loss of the fibers at the wavelength region of interest (700-1200 nm in air, 350-600 nm in isopropanol) increases with the concentration of NPs in the impregnation solution. Strikingly, the losses are below $9 \mathrm{~dB} \mathrm{~m}^{-1}$ using a $1.8 \times 10^{17} \mathrm{NPs} \mathrm{L}^{-1}$ impregnation solution for the medium-sized NPs. These values are considerably lower than those reported in our previous work of in situ growth of Rh NPs in the fibers [20]. Furthermore, the size of the NPs also influences the light transmission, obtaining higher losses with increasing NP diameters at the same impregnation solution concentration. Nevertheless, it must be noted that the loss introduced by the biggest particles tested is around $15 \mathrm{~dB} \mathrm{~m}^{-1}$. It means a $5 \mathrm{~dB}$ loss for the typical fiber length used in the experiments using $35 \mathrm{~cm}$ fiber, which allows sufficient transmission for in situ catalysis monitoring.

((Figure 3))

\subsection{Stable PtNi NPs deposition onto HC-PCFs}

Firstly, it needs to be noted that in line with the results obtained using the conventional reactor, the evolution of the conversion of DR1 in the fiber was also found to follow a pseudo secondorder kinetic model (see exemplary curves in Fig. 4). Therefore, here, the activity of the HCPCF microreactor within the hydrogenation of DR1 is used as a benchmark to obtain an “apparent” catalyst concentration. It is due to the low volume of the HC-PCF microreactor, which is in the range of $\mathrm{nL} \mathrm{cm} \mathrm{cm}^{-1}$, the immobilized amount of NPs is difficult to determine e.g. by classical CO-Chemisorption or ICP methods. The kinetic obtained in the conventional tank reactor under the same operating conditions and with known concentration and number of 
catalyst NPs is used as reference (see Fig. S6 for the calibration curve correlating activity to catalyst concentration). The apparent concentration of NPs is the concentration, which would be needed to obtain in the tank reactor the same activity as observed experimentally within the HC-PCF microreactor.

((Figure 4))

With this approach, the resulting apparent catalyst concentration determined through the initial catalytic activity of the fibers were observed to strongly depend on the impregnation time as depicted in Fig. S7. In this sense, fibers prepared using fifteen-minute impregnation times exhibited the highest starting loading with NPs in the fiber. Nevertheless, for longer time on streams (e.g. $240 \mathrm{~min}$ ) it became obvious that the activity drops strongly (e.g. by $\sim 50 \%$ ). Thus, it seems that not all initially deposited NPs are stable immobilized and weakly bonded NPs are lost during a long-time study. To investigate the stability of the NPs attachment, the fibers with the maximum apparent number of clusters after 15 min of impregnation time were continuously flushed with isopropanol $\left(10 \mu \mathrm{L} \mathrm{min}{ }^{-1}\right)$ for several hours. Interestingly, the flushing resulted in a notable reduction of the activity and approximately $90 \%$ of the active sites washed away from the internal core of the fiber. This suggests that after the immobilization two types of NPs are present inside of the fiber. Weakly attached NPs, which are easily dragged by the isopropanol flow and strongly attached ones, which remain even after long time flushing. The strongly attached NPs are most likely those who have a direct interaction with the fiber surface, and their adhesion forces can be estimated to be in the range of $10^{-9}$ Newtons [36, 37], which are well above the drag force ( $\sim 0.001 \mathrm{nN}$ ) experienced by the NPs close to the HC-PCF surface during the flow. As a result, after flushing an apparent constant catalyst concentration in the fibers of approx. $7.3 \mathrm{mg} \mathrm{L}^{-1}$ results, more or less independent of the initial NPs concentration (see Fig. S8).

These findings represent a backdrop in the preparation of reliable catalytic HC-PCF microreactors, where only strongly attached NPs are present and thus reliable catalytic results 
can be obtained. For a reproducible preparation of catalytic PCF microreactors it is therefore suggested to deposit initially an excess number of NPs allowing to fill all strong anchor sides. Subsequently, weakly attached NPs need to be flushed out. For the NPs used within this study this is achieved with an impregnation time of fifteen-minutes and a subsequent five-hours isopropanol flushing. While for the different preparation methods presented in Fig. S8 the number of particles deposited varied by a factor of $\sim 40$, with the new method and for different fibers an average number of $4.43 \times 10^{15} \mathrm{NPs} \mathrm{L}^{-1}$ with a very low standard deviation of $0.46 \times$ $10^{15}$ NPs L ${ }^{-1}$ could be achieved. Remarkably, it is important to highlight that the total amount of PtNi catalyst used to prepare the tiny HC-PCF microreactors for similar kinetic results as in the classical tank reactor, is six orders of magnitude lower $\left(\sim 1 \times 10^{-9}\right.$ vs. $1 \times 10^{-3} \mathrm{~g}$ of $\left.\mathrm{Pt}\right)$, highlighting the major opportunities that HC-PCF microreactors offer in low-cost screening of novel catalysts..

To get additional insights to the results obtained with $6.3 \mathrm{~nm}$ NPs also 4.4 and $8.3 \mathrm{~nm}$ PtNi bimetallic NPs were immobilized in HC-PCF microreactors following the new method. Fig. 5 depicts the apparent number of NPs calculated from the catalytic experiments (see values in Tab. 1). For 6.3 and $8.3 \mathrm{~nm}$ particles, an apparent similar amount in the range of 4.5 to $5.8 \times 10^{15} \mathrm{NPs} \mathrm{L}^{-1}$ were deposited. Interestingly, the $4.4 \mathrm{~nm}$ particles could be immobilized with an approx. 2.5 higher particle density. Remarkably, this higher NP density did not affect the light transmission of the fiber, and optical losses of the isopropanol-filled HC-PCFs were as low as $2 \mathrm{~dB} \mathrm{~m}^{-1}$. Increasing the NPs size increased the losses, despite the lower number of NPs present $\left(6.3 \mathrm{~nm}=\sim 19 \mathrm{~dB} \mathrm{~m} \mathrm{~m}^{-1}\right.$ and $8.3 \mathrm{~nm}=\sim 24 \mathrm{~dB} \mathrm{~m}^{-1}$ in isopropanol-filled HC-PCFs), confirming that the optical losses are mostly governed by the size of the immobilized NPs. Thus, in the range of interest for catalysis, which is approx. below $10 \mathrm{~nm}$, the optical properties of the resulting HC-PCF microreactors are sufficient to carry out in situ spectroscopic experiments. Especially for smaller particles $<5 \mathrm{~nm}$ the scattering losses reduce strongly, which would allow to use even meter-long HC-PCF microreactors for increasing 
spectroscopic sensitivity. Finally, to test the stability of the immobilization after the new procedure, kinetic experiments were carried out with an as prepared HC-PCF microreactor and after an additional $50 \mathrm{~h}$ flushing with isopropanol. During this extended period, the catalytic activity dropped only by slightly by $15 \%$.

((Figure 5))

((Table 1))

\section{Conclusion}

It has been developed a method for the stable immobilization of well-defined PtNi bimetallic catalyst NPs inside HC-PCF microreactors for in situ catalysis monitoring, while the sizes are in the range of interest for catalytic studies $(<10 \mathrm{~nm})$. These novel ex situsynthesized and size-controlled PtNi NPs were demonstrated to remain highly attached into the fiber for long-term experiments. They also, for small sizes, reduced the scattering losses compared to previous methods of in situ growth of particles inside the fibers. In addition, the measured catalytic activity in the fibers was found to be in good agreement with the results obtained in the conventional tank reactor. Remarkably, the tiny HC-PCF microreactor requires six orders of magnitude less amount of precious catalyst to obtain kinetic information as a conventional tank reactor. For these reasons, this new generation of HC-PCF microreactors could offer unprecedented opportunities in catalyst screening for structure sensitive, and long-term reactions.

\section{Acknowledgements}

The authors gratefully acknowledge the funding of the German Research Council (DFG) which, within the framework of its 'Excellence Initiative', supports the Cluster of Excellence 'Engineering of Advanced Materials' (www.eam.uni-erlangen.de) at the University of Erlangen-Nuremberg. The authors are also grateful to Eduard Butzen and Haitham Salti for collaboration. 


\section{Symbols}

$L$

$n$
Fiber length

Refractive index [cm]

$[-]$

\section{Abbreviations}

Beam splitter

(CCD)

Charge coupled device

(DR1)

Disperse Red 1

(HC-PCF)

Hollow-core photonic crystal fiber

(NPs)

Nanoparticles

(OSA)

Optical spectrum analyzer

(SI)

Supporting information

$\mathrm{UV} / \mathrm{Vis}$

Ultraviolet/Visible

\section{References}

[1] J. Hagen, Industrial catalysis: a practical approach, John Wiley \& Sons, 2015.

[2] M. Sankar, N. Dimitratos, P. J. Miedziak, P. P. Wells, C. J. Kiely, G. J. Hutchings, Chem. Soc. Rev., 2012, 41 (24), 8099-8139.

[3] T. Wirth, Microreactors in organic chemistry and catalysis, John Wiley \& Sons, 2013.

[4] E. E. Coyle, M. Oelgemöller, Photochem. Photobiol. Sci., 2008, 7 (11), 1313-1322.

[5] N. Al-Rifai, E. Cao, V. Dua, A. Gavriilidis, Current Opinion in Chemical Engineering, 2013, 2 (3), 338-345.

[6] X. Fan, I. M. White, Nature photonics, 2011, 5 (10), 591-597.

[7] A. R. Hawkins, H. Schmidt, Handbook of optofluidics, CRC Press, 2010.

[8] J. Parmar, S. Jang, L. Soler, D.-P. Kim, S. Sánchez, Lab Chip, 2015, 15 (11), 23522356.

[9] M. Schmidt, A. M. Cubillas, N. Taccardi, T. G. Euser, T. Cremer, F. Maier, H. P. Steinrück, P. St. J. Russell, P. Wasserscheid, B. J. M. Etzold, ChemCatChem, 2013, 5 (3), 641-650.

[10] A. M. Cubillas, S. Unterkofler, T. G. Euser, B. J. M. Etzold, A. C. Jones, P. J. Sadler, P. Wasserscheid, P. St. J. Russell, Chem. Soc. Rev., 2013, 42 (22), 8629-8648.

[11] P. St. J. Russell, Science, 2003, 299 (5605), 358-362.

[12] P. St. J. Russell, J. Lightwave Technol., 2006, 24 (12), 4729-4749. 
[13] A. M. Pinto, M. Lopez-Amo, Journal of Sensors, 2012, 2012.

[14] T. Ritari, J. Tuominen, H. Ludvigsen, J. Petersen, T. Sørensen, T. P. Hansen, H. R. Simonsen, Opt. Express, 2004, 12 (17), 4080-4087.

[15] J. S. Chen, T. G. Euser, N. J. Farrer, P. J. Sadler, M. Scharrer, P. St. J. Russell, Chem. Eur. J., 2010, 16 (19), 5607-5612.

[16] G. O. Williams, J. S. Chen, T. G. Euser, P. St. J. Russell, A. C. Jones, Lab Chip, 2012, 12 (18), 3356-3361.

[17] A. Khetani, M. Laferrière, H. Anis, J. Scaiano, J. Mater. Chem., 2008, 18 (40), 47694774.

[18] S. Unterkofler, R. J. McQuitty, T. Euser, N. J. Farrer, P. Sadler, P. St. J. Russell, Opt. Lett., 2012, 37 (11), 1952-1954.

[19] A. M. Cubillas, M. Schmidt, M. Scharrer, T. G. Euser, B. J. M. Etzold, N. Taccardi, P. Wasserscheid, P. St. J. Russell, Chem. Eur. J., 2012, 18 (6), 1586-1590.

[20] A. M. Cubillas, M. Schmidt, T. G. Euser, N. Taccardi, S. Unterkofler, P. St. J. Russell, P. Wasserscheid, B. J. M. Etzold, Adv. Mater. Interfaces, 2014, 1 (5).

[21] W. Zang, G. Li, L. Wang, X. Zhang, Catal. Sci. Technol., 2015, 5 (5), 2532-2553.

[22] H. Yan, C. Gu, C. Yang, J. Liu, G. Jin, J. Zhang, L. Hou, Y. Yao, Appl. Phys. Lett., 2006, 89 (20), 204101.

[23] Y. Zhang, C. Shi, C. Gu, L. Seballos, J. Z. Zhang, Appl. Phys. Lett., 2007, 90 (19), 193504.

[24] J. Baeza, L. Calvo, M. Gilarranz, A. Mohedano, J. Casas, J. Rodriguez, J. Catal., 2012, 293, 85-93.

[25] J. Baeza, L. Calvo, M. Gilarranz, J. Rodriguez, Chem. Eng. J., 2014, 240, 271-280.

[26] R. Ferrando, J. Jellinek, R. L. Johnston, Chem. Rev., 2008, 108 (3), 845-910.

[27] Z. Dong, X. Le, C. Dong, W. Zhang, X. Li, J. Ma, Appl. Catal., B, 2015, 162, 372-380.

[28] M. Munoz, Z. M. de Pedro, J. A. Casas, J. J. Rodriguez, Appl. Catal., A, 2014, 488, 78-85.

[29] N. A. Bakar, M. Bettahar, M. A. Bakar, S. Monteverdi, J. Ismail, M. Alnot, J. Catal., 2009, 265 (1), 63-71.

[30] J. Li, W.-P. Tian, L. Shi, Catal. Lett., 2011, 141 (4), 565-571.

[31] G. Liang, L. He, M. Arai, F. Zhao, ChemSusChem, 2014, 7 (5), 1415-1421.

[32] W. Yu, M. D. Porosoff, J. G. Chen, Chem. Rev., 2012, 112 (11), 5780-5817.

[33] K. Sahel, N. Perol, H. Chermette, C. Bordes, Z. Derriche, C. Guillard, Appl. Catal., B, 2007, 77 (1), 100-109.

[34] M. Munoz, S. Ponce, G.-R. Zhang, B. J. M. Etzold, Appl. Catal., B, 2016, 192, 1-7.

[35] G. Antonopoulos, F. Benabid, T. Birks, D. Bird, J. Knight, P. St. J. Russell, Opt.

Express, 2006, 14 (7), 3000-3006.

[36] Q. K. Ong, I. Sokolov, J. Colloid Interface Sci., 2007, 310 (2), 385-390.

[37] W. Rong, A. E. Pelling, A. Ryan, J. K. Gimzewski, S. K. Friedlander, Nano Lett., 2004, 4 (11), 2287-2292. 
Table 1. Impregnation times, rate constants and calculated apparent number of catalysts present in HC-PCF microreactors loaded with an immobilization solution of $1.2 \times 10^{17} \mathrm{NPs}^{-}$ 1 .

\begin{tabular}{|c|c|c|c|c|c|c|}
\hline Denomination & $\begin{array}{c}\text { Impregnation } \\
\text { time } \\
{[\mathrm{s}]}\end{array}$ & $\begin{array}{c}\text { Flow time } \\
\text { [h] }\end{array}$ & $\begin{array}{c}\text { NPs size } \\
\text { [nm] }\end{array}$ & $\begin{array}{c}k^{،} \\
{\left[\mathrm{~L} \mathrm{umol}^{-1} \mathrm{~s}^{-1}\right]}\end{array}$ & $\begin{array}{c}\text { Apparent catalysts } \\
\text { concentration } \\
{\left[\mathrm{mg} \mathrm{L}^{-1}\right]}\end{array}$ & $\begin{array}{c}\text { Apparent \#NPs per liter } \\
\left.\qquad 10^{15} \mathrm{NPs} \mathrm{L}^{-1}\right]\end{array}$ \\
\hline 6.nm-HC1-0 & $<3$ & 0 & 6.3 & 0.000138 & 5.0 & 3.04 \\
\hline 6.nm-HC2-0 & 300 & 0 & 6.3 & 0.000925 & 20.2 & 12.30 \\
\hline 6.nm-HC3-0 & 900 & 0 & 6.3 & 0.005460 & 58.5 & 35.52 \\
\hline 6.nm-HC3-5 & 900 & 5 & 6.3 & 0.000220 & 7.3 & 4.43 \\
\hline 6.nm-HC3-50 & 900 & 50 & 6.3 & 0.000178 & 6.2 & 3.77 \\
\hline 4.4nm-HC3-5 & 900 & 5 & 4.4 & 0.000347 & 12.0 & 18.00 \\
\hline 8.3nm-HC3-5 & 900 & 5 & 8.3 & 0.000152 & 43.0 & 5.81 \\
\hline
\end{tabular}

\section{Figure Legends}

Figure 1. (a) SEM image of the kagomé HC-PCF used in the experiments, (b) TEM image of $6.3 \mathrm{~nm}$ PtNi NPs, and (c) Optical setup used for the DR1 hydrogenation in particle-deposited kagomé HC-PCFs. Inset left shows the side-view of the irradiated kagomé HC-PCF. Inset right shows the guided mode in the core, when the fiber is filled with isopropanol and impregnated with $6.3 \mathrm{~nm}$ PtNi NPs. MMF: multimode fiber.

Figure 2. Effect of PtNi-6.3 concentration on DR1 hydrogenation in a conventional stirredtank reactor. Experimental (symbols) and predicted (lines) values.

Figure 3. Average loss at $600 \mathrm{~nm}$ of the air-filled fibers impregnated with different immobilization solution concentration of NPs following the standard method (lines guide the eye).

Figure 4. (a) Example of conversion vs. time curves of a HC-PCF (6.3 nm NPs, impregnation time: 5 min without flushing) and a batch reactor $\left(6.3 \mathrm{~nm} \mathrm{NPs}\right.$ and $6.5 \mathrm{mg} \mathrm{L}^{-1}$ catalysts concentration), and (b) Example fitting of the hydrogenation of DR1 in HC-PCFs (6.3 nm 
NPs, impregnation time: 5 min without flushing) compared to a batch reactor (6.3 $\mathrm{nm}$ and 6.5 $\mathrm{mg} \mathrm{L}^{-1}$ catalysts concentration) to a second order reaction kinetic model.

Figure 5. Apparent number of clusters present in the inner core, and optical losses in isopropanol-filled HC-PCFs with different bimetallic NP sizes.
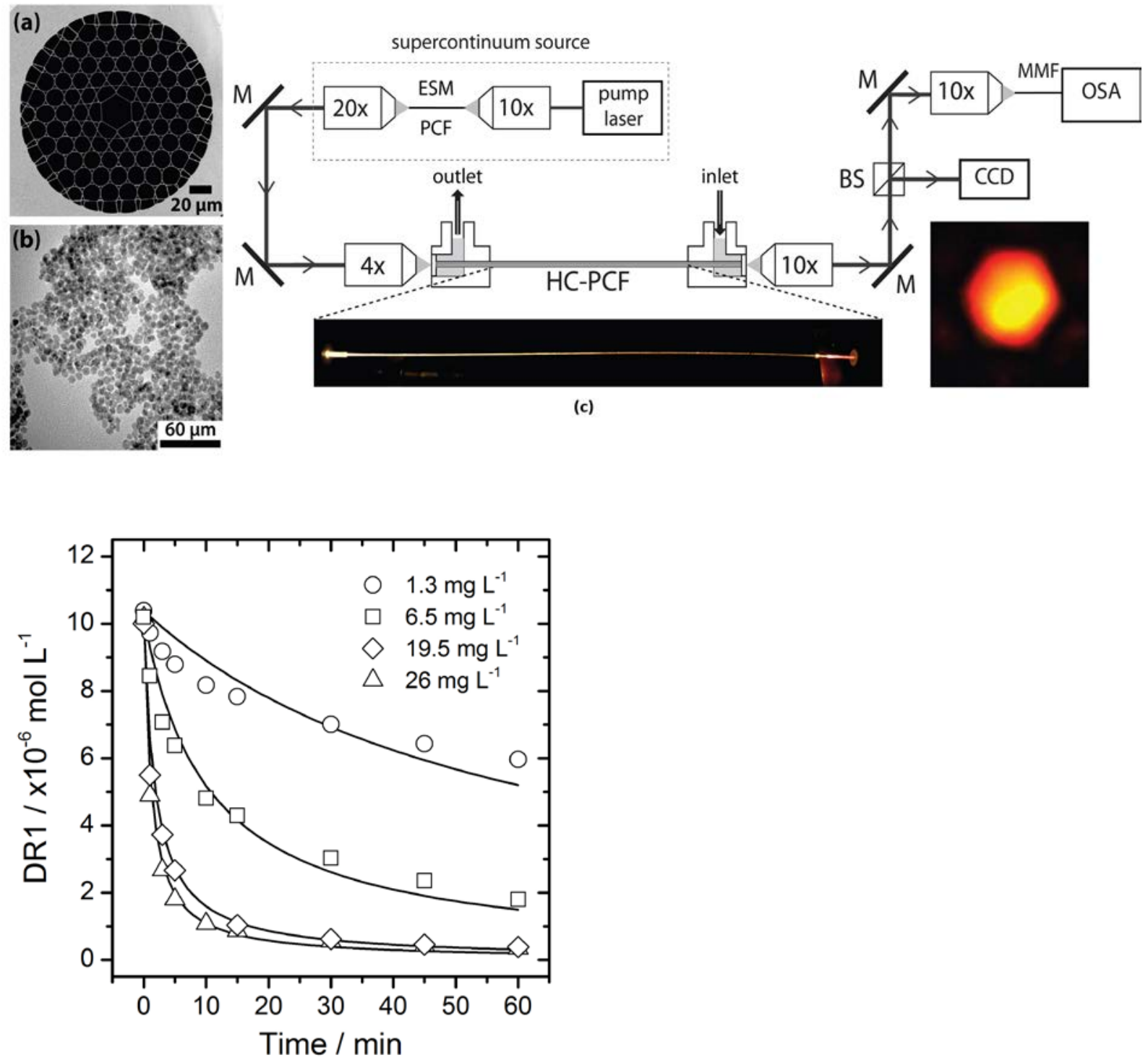


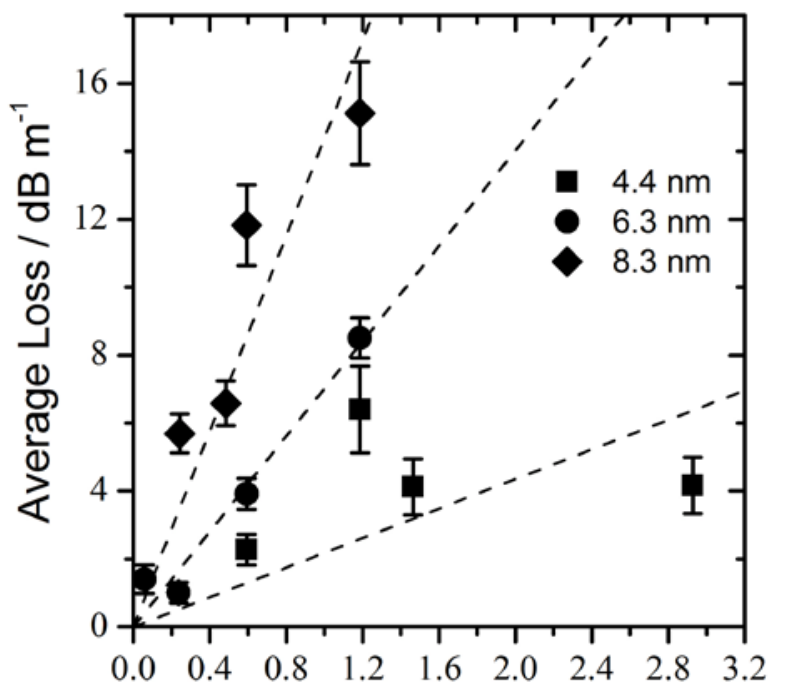

Impregnation Solution Concentration / $\times 10^{17} \mathrm{NPs} \mathrm{L}^{-1}$
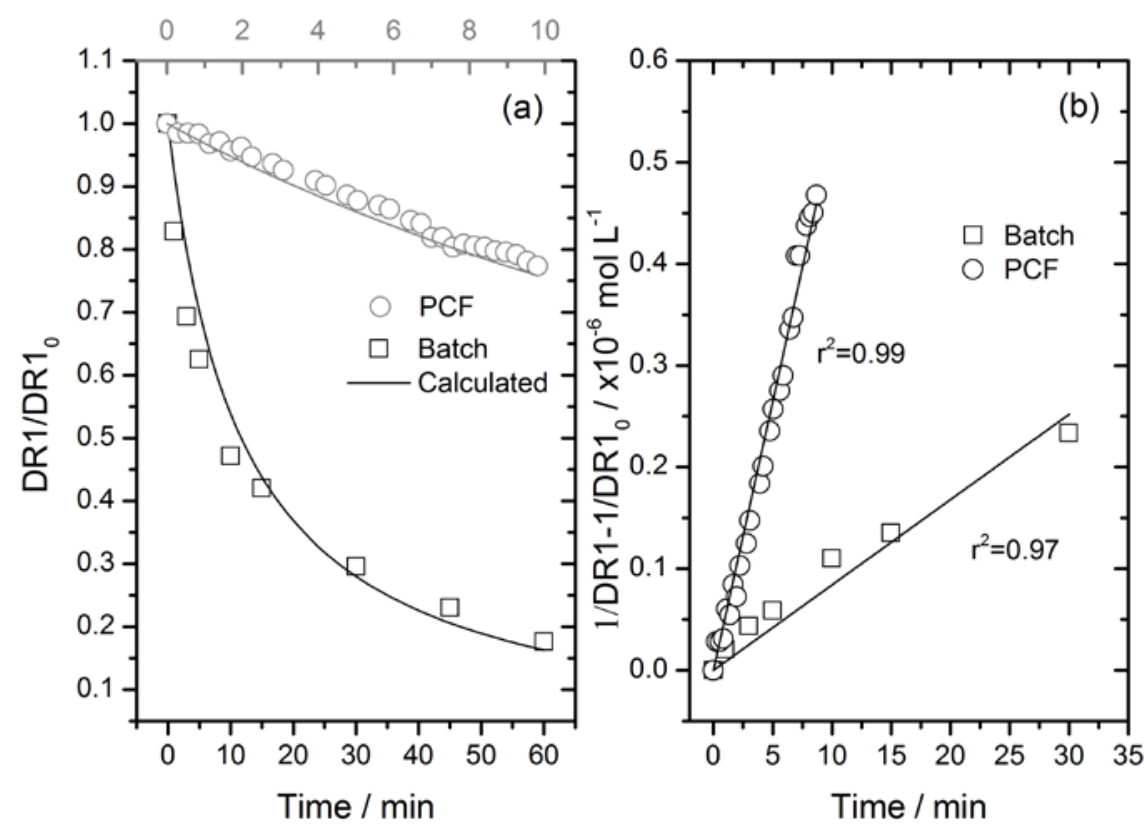


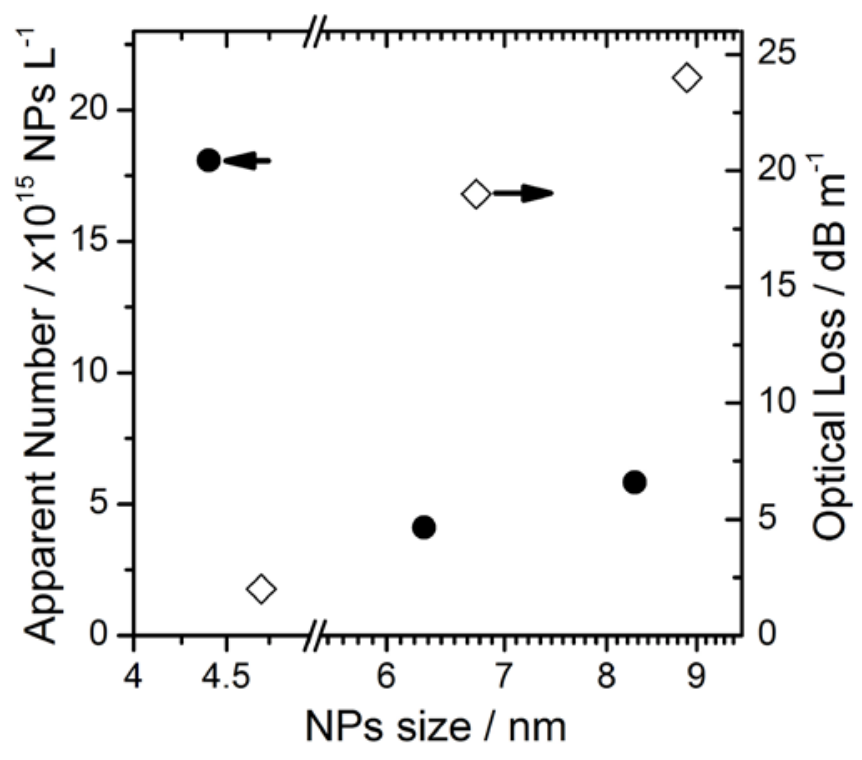

Text for the Table of Contents (TOC)

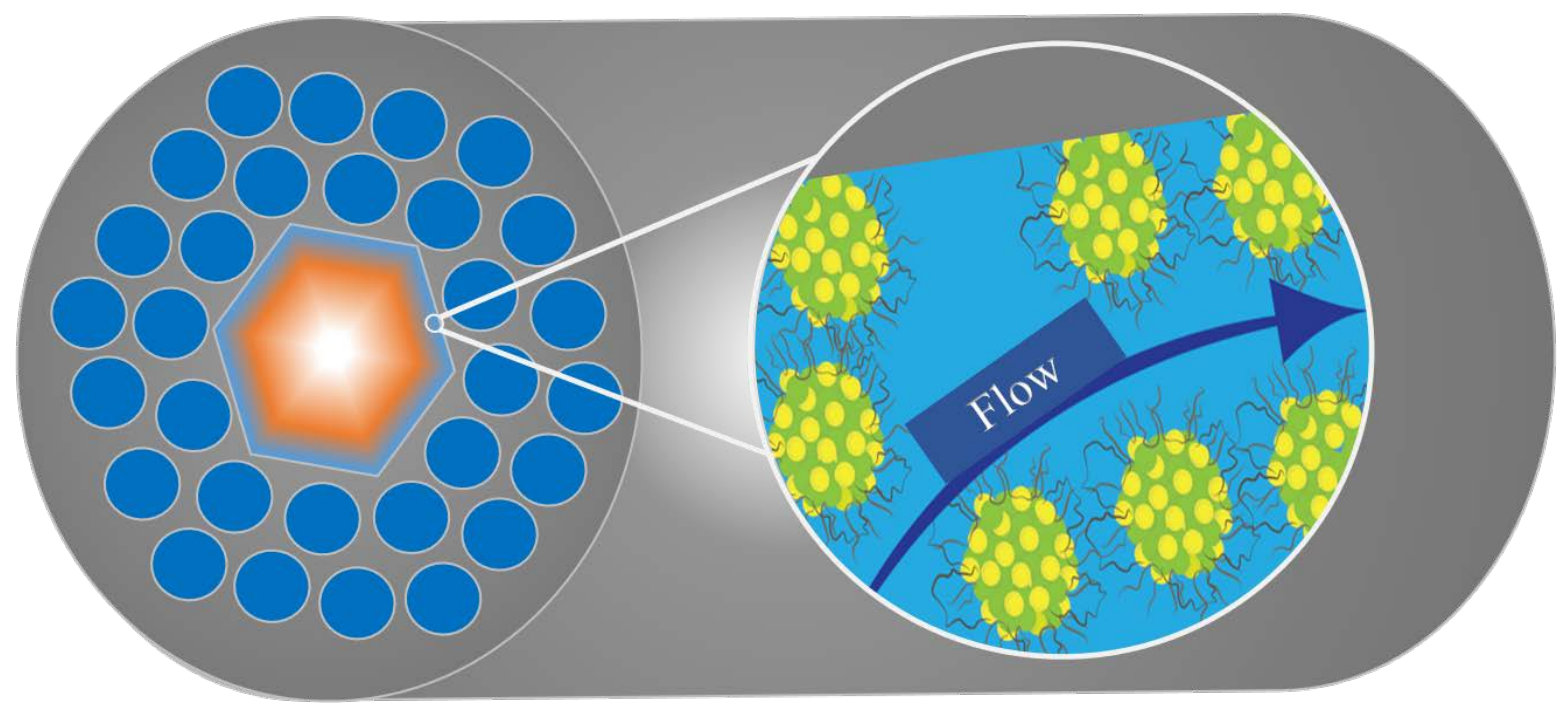

